\title{
PARP Inhibitor CEP-9722
}

National Cancer Institute

\section{Source}

National Cancer Institute. PARP Inhibitor CEP-9722. NCI Thesaurus. Code C84880.

A small-molecule prodrug of CEP-8983, a novel 4-methoxy-carbazole inhibitor of the nuclear enzymes poly(ADP-ribose) polymerase (PARP) 1 and 2, with potential antineoplastic activity. Upon administration and conversion from CEP-9722, CEP-8983 selectively binds to PARP 1 and 2, preventing repair of damaged DNA via base excision repair (BER). This agent enhances the accumulation of DNA strand breaks and promotes genomic instability and apoptosis. CEP-8983 may potentiate the cytotoxicity of DNAdamaging agents and reverse tumor cell chemo- and radioresistance. PARP catalyzes post-translational ADP-ribosylation of nuclear proteins that signal and recruit other proteins to repair damaged DNA and can be activated by single strand breaks in DNA. 